\title{
Hörgeräte können kognitiven Abbau bremsen
}

\section{Eine aufwändige Studie über 25 Jahre zeigt einen Zusammenhang zwischen Schwerhörigkeit und kognitivem Verfall. Mit Hörgerät blieben die Betroffenen geistig länger fit.}

ᄃ ür eine Langzeitstudie untersuchten Forscher aus Frankreich in einer $\mathrm{Zu}$ fallsstichprobe circa 3.700 Probanden

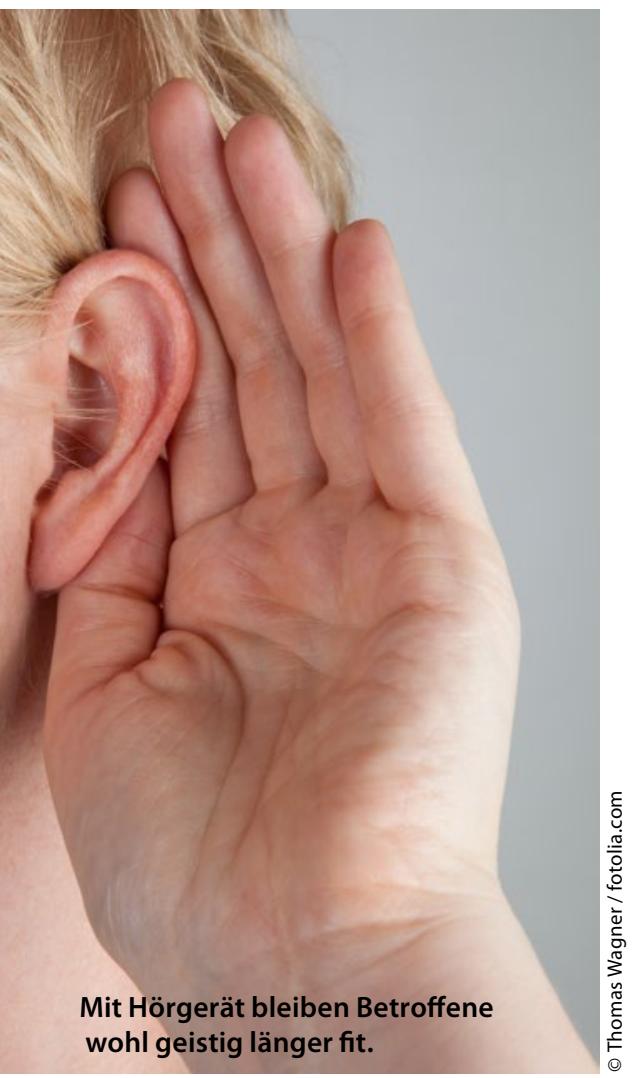

im Alter $\geq 65$ Jahren in 2- bis 3-jährigen Abständen über insgesamt 25 Jahre hinweg. Sie testeten sie dabei auf geistige Leistungsfähigkeit, Depressivität, Alltagsselbstständigkeit und Sozialkontakte. Zu Studienbeginn Ende der 1980erJahre wurden die Probanden auch nach ihrem Gehör gefragt und in drei Gruppen klassifiziert: Keine $(n=2.394)$, leichtbis mittelgradige $(\mathrm{n}=1.139)$ und schwere $(\mathrm{n}=137)$ Hörbeeinträchtigung. Ca. $8 \%$ der leicht- bis mittelgradig und $45 \%$ der hochgradig Schwerhörigen waren mit einem Hörgerät versorgt.

Mit diesem Studiendesign konnten die Auswirkungen von Schwerhörigkeiten auf die kognitiven Fähigkeiten der Probanden auch in Abhängigkeit vom Tragen eines Hörgeräts über einen langen Zeitraum untersucht werden.

Nach 25 Jahren erreichten die hörbeeinträchtigten Probanden schlechtere kognitive Testergebnisse - allerdings nur jene ohne Hörgerät. Mit Hörgerät verlief der kognitive Abbau nicht rascher als der von Normalhörenden. Die Geräte scheinen die Kognition positiv zu beeinflussen, da kommunikative Fähigkeiten verbessert, soziale Aktivitäten gefördert und depressive Verstimmungen verhindert werden.

Die Autoren schließen, dass Schwer- hörigkeit mit einem beschleunigten geistigen Abbau einhergeht und dass das Tragen von Hörgeräten diese Beschleunigung zurücknehmen kann.

Amieva H, Ouvrard C, Giulioli C et al. Self-reported hearing loss, hearing aids, and cognitive decline in elderly adults: a 25-year study. J Am Geriatr Soc. 2015;63:2099-104

\section{Kommentar}

Diese Studie ist wegen ihrer Größe und ihrer langen Beobachtungsdauer bemerkenswert. Erstmals wird hier gezeigt, dass der Gebrauch von Hörgeräten den bei Hörgeschädigten zu beobachtenden rascheren kognitiven Abbau verlangsamen kann. Eine subtile statistische Analyse legt nahe, dass dieser positive Effekt dadurch zustande kommt, dass Depressivität und soziale Isolation bei Hörgeräteträgern seltener sind. Die Untersuchung war als Beobachtungsstudie angelegt und hatte kein randomisiertes, kontrolliertes Studiendesign. So ist sie zwar letztlich nicht beweisend dafür, kann aber trotzdem als starker Hinweis darauf angesehen werden, dass der alterungsbedingte kognitive Verfall bei Hörgeschädigten rascher verläuft - und man sich mit Hörgeräten davor schützen kann.

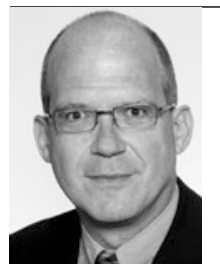

Dr. med. Joachim Zeeh Facharzt für Innere Medizin Klinische Geriatrie Palliativmedizin Geriatrische Fachklinik Georgenhaus 98617 Meiningen

\section{Neue Keime - größere Gefahr}

Alle reden über MRSA, inzwischen werden allerdings andere antibiotikaresistente Keime zu einem größeren Problem.

W ährend viel über den Methicillinresistente Staphylococcus aureus (MRSA) und gramnegative Erreger gesprochen wird, möchte Prof. Dr. Gerd Fätkenheuer, Köln, das Augenmerk auf Vancomycin-resistente Enterokokken (VRE) lenken: „Sie sind insofern ein gröBeres Problem als MRSA, weil uns viel weniger Medikamente zur Verfügung stehen." Bei den gramnegativen Erregern haben u.a. Massentierzucht und internationale Reisetätigkeit eine Zunahme von Bakterien, die Extended-Spectrum-Betalaktamasen (ESBL) bilden, verursacht. Von fast 600 Tropenreisenden kehrte einer französischen Studie zufolge die Hälfte mit multiresistenten Erregern im Darm zurück [Clin Infect Dis.
2015; 61:593-600]. „Das zeigt, dass in Indien, China und Afrika die Verbreitung bereits so groß ist, dass der Kontakt damit gar nicht zu vermeiden ist", so Fätkenheuer.

Das Bewusstsein dafür, dass Patienten mit Infektionskrankheiten besser versorgt werden müssen, scheint zu wachsen. Sogar bei einem G7-Gipfel war die Problematik bereits Thema. 